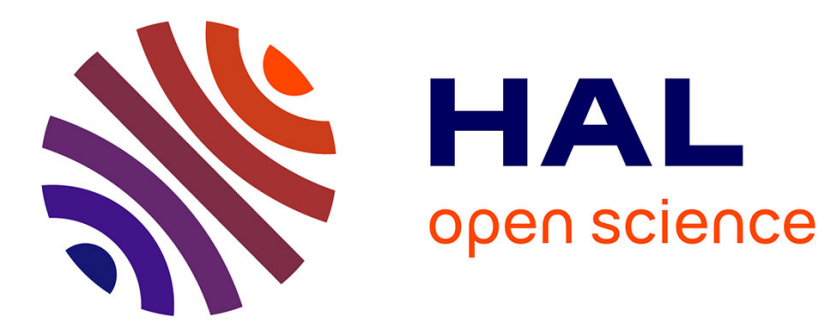

\title{
Radar simulation in SiVIC platform for transportation issues. Antenna and propagation channel modelling
}

Steve Pechberti, Dominique Gruyer, Vincent Vigneron

\section{To cite this version:}

Steve Pechberti, Dominique Gruyer, Vincent Vigneron. Radar simulation in SiVIC platform for transportation issues. Antenna and propagation channel modelling. 15th International IEEE Conference on Intelligent Transportation Systems (ITSC 2012), Sep 2012, Anchorage, United States. pp.469-474, 10.1109/ITSC.2012.6338631 . hal-00870879

\section{HAL Id: hal-00870879 \\ https://hal.science/hal-00870879}

Submitted on 5 Jul 2021

HAL is a multi-disciplinary open access archive for the deposit and dissemination of scientific research documents, whether they are published or not. The documents may come from teaching and research institutions in France or abroad, or from public or private research centers.
L'archive ouverte pluridisciplinaire HAL, est destinée au dépôt et à la diffusion de documents scientifiques de niveau recherche, publiés ou non, émanant des établissements d'enseignement et de recherche français ou étrangers, des laboratoires publics ou privés. 


\title{
Radar simulation in SiVIC platform for transportation issues. Antenna and propagation channel modelling*
}

\author{
Steve PECHBERTI ${ }^{1}$, Dominique GRUYER ${ }^{1}$ and Vincent VIGNERON ${ }^{3}$
}

\begin{abstract}
This paper proposes a new radar sensor modelling for Advanced Driver Assistance Systems (ADAS) prototyping. The model is embedded on the SiVIC platform (Simulator for Vehicle, Infrastructure and Sensors). Lots of simulators already exist for this issue, but none is designed to address the objectives of real-time computation, highly sampled signal generation. And few simulators offer the ability to be integrated in a dynamic platform for the ADAS prototyping. In this paper, several radar technologies will be presented. Then, a radar designed especially for automotive domain will be described exploring each subparts, radar antenna andi.e. propagation channel. Such as the generic model, hypothesis done on electromagnetic waves and environmental objects modelling will also be provided. A first model of simple duplex radar with Frequency Shift Keying (FSK) modulation is implemented and shown as illustration for the defined architecture. Finally, in order to optimize the duration for signal generation, several software architecture solution will be proposed.
\end{abstract}

\section{INTRODUCTION}

Many driver assistance systems are studied to improve the safety of road environments. An ego vehicle perception and the corresponding reaction of the vehicle are generally taken into account. However, ego perception is not satisfactory in many cases. Risk must be minimized and driving security can be improved by adding information. The resources needed are however time-consuming and expensive. It therefore becomes essential to have a simulation environment, to enrich the prototyping and the cooperative driving assistance systems in the early stage of the system design.

In this article, we describe the long range radar, the most popular sensor in Advanced Driver Assistance Systems (ADAS) prototyping. This model has been developped and integrated in SiVIC [8].

The radar model presented here has two main objectives:

1) to provide accurate data about radar target detection, to be integrated in the simulation loop during ADAS prototyping.

2) to obtain a realistic radar model. This means that the model will take into account the antenna physics and its uncertainties.

The model is sufficiently accurate and mature to be used as a research tool for radar analysis and to evaluate new signal processing algorithms.

\footnotetext{
*This work was supported by France-Quebec CooPERCOM ANR program.

${ }^{1}$ S. Pechberti and D. Gruyer are with the LIVIC laboratory, IFSTTAR, 14 Route de la minière, 78000 Versailles, France \{steve.pechberti, dominique.gruyer\} @ifsttar.fr

${ }^{3} \mathrm{~V}$. Vigneron is with the IBISC laboratory, UEVE, 40, Rue du Pelvoux, 91020 Evry-Courcouronnes, France - vvignediup. univ-evry. fr
}

\section{STATE OF THE ART}

\section{A. Available electromagnetic simulator}

In issues related to simulation of radar sensors, many solutions have resulted in commercial products, usually in medical, telecommunication, or environment perception ${ }^{1}$ domains.

ENDOCOM for instance is designed to study the influence of electromagnetic phenomenons in the human body [2]. Most of these simulators are based on statistical modeling [9]. This approach is good enough for the purpose. But statistical modeling is well designed for static environment where some parameters only could evolved. In the case of complex dynamic urban scenarii, it is not possible to conceive a compliant modeling which could be suitable for all kind of situations. Statistics are not adapted to this kind of transportation applications.

Some others approaches such as MultiPASS [3] and OP2H [4] for instance are designed to study radiation exposure in town or to evaluate the implementation of new telecommunication antennas in urban environment. Unfortunately, these simulators focused again on static situations. Nevertheless, it is even possible, under some specific conditions, to get a dynamic behavior, but not in real time. Moreover, these simulators are for city level applications whereas our researches deal with vehicle level applications.

Few works really study radar in automotive scenarii [7]: these simulators are named WinPROP[10], FERMAT[1]. They provide good approximations for the purpose they served but they can not be used in real time scenario simulation for ADAS prototyping. And these radar simulations do not provide signal outputs for online or offline processing.

\section{B. Radar zoology}

Differents technologies are available and have been implemented for radar application. We can classify them into 3 main categories:

a) Pulse Radars: They periodically emit a very short pulse. The measured distance is deduced from the delay between the received signal and the emitted one. Remarkable properties for this type of technology are the pulse duration $\tau$ andthe Pulse Repetition Frequency (PRF) $f_{r}$ and the Pulse Repetition Rate (PRR).

This type of radar is not compliant with automotive application. Indeed, the shorter distance that can be computed relies on the PRF and follows the equation: $D=\frac{c P R F}{2}$. It means

\footnotetext{
${ }^{1}$ especially for transportation and military application.
} 
that for detecting a 3 meters distance target, we need a PRF of $133 \mathrm{MHz}$, which corresponds to a duration of $7.510^{-} 9$ $\mathrm{s}$, which suposes an expensive hardware. For the automotive domain, this kind of system is not relevant.

b) Continuous Waves Radars $(\mathrm{CW})$ : These radars continously emit an electromagnetic wave. This electromagnetic wave can be either monochromatic or modulated. For monochromatic waves, we only have access to the target velocity. This technology is typically used in competition sports like golf and tennis or in speed enforcement camera where only the over speed is important. Is is however possible to have access of both information, distance and velocity, by combinating two $\mathrm{CW}$ radars with low frequency gap, or by using a modulated signal, such as in FMCW radars.

The phase difference gives the target distance by: $\Delta \Phi=$ $\frac{4 . \pi . \Delta f}{c}$, where $c$ is the light speed and $\Delta f$ the frequency difference between the two signals. The velocity of the target is defined by the doppler frequency of one of the signals.

The two categories are usable for automotive domain. In this paper, we will focus on the second category by considering the FSK modulation.

\section{GENERIC RADAR MODELING}

\section{A. The general architecture}

The radar sensor modeling can be seen as two distinct issues. The first issue deals with the hardware modelisation, namely the radar antenna, whereas the second issue deals with the wave propagation in the environment (propagation channel).

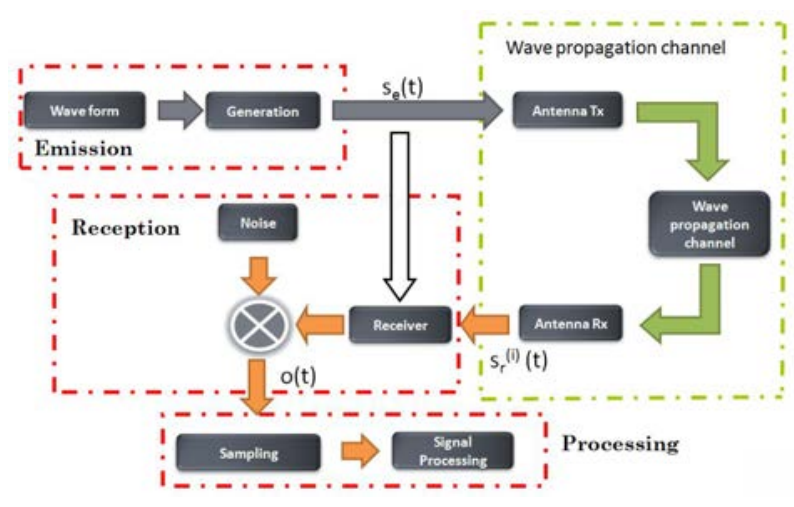

Fig. 1. General architecture for electromagnetic sensor modeling

The Figure 1 presents the chosen model cutting. The left part (dashed red) shows the radar antenna model; the right part (dashed green) focuses on the electromagnetic wave propagation channel model.

\section{B. The Radar Antenna Modeling}

The radar antenna simulation is defined by three distinct modules. Each one simulates a specific functionnality of the sensor. The three module are

- the transmission module,

- the reception module,
- the processing module.

In order to take into account physical hardware imperfections, the antenna modeling involves

- hardware specifications to simulate internal noises and gains,

- the antenna diagram used for transmission pattern.

These information are obtained thanks to manufacturers or by specific experiments.

The generic model (1) defined above provides for the antenna model, a generic architecture for the simulator which allow to easily integrate new radar modelling or new specific signal processing. It is well suited for all kind of radar antenna. Typically, for a specific modulation, only the transmission module and processing module have to be adapted. The receiver module is applicable for all types of antenna. It is also easy to switch only the processing module in order to test new radar algorithms.

1) The transmission module: It generates the source modulated signal which is spreaded in virtual scene. As signal is periodic and modulated, it is not necessary to proceed to the generation for each iteration of the simulation. The job is done once only during simulation, except if parameters about the wave form or signal are modified. The signal generation depends on the type of radar. Its implementation is specific for each of antenna modeling. The emitted wave is continuous, and can be defined by:

$$
\begin{aligned}
& \text { in } \mathbb{R}, \quad s_{e}(t)=A(t) \cos (\omega(t) t+\phi(t)) \\
& \text { in } \mathbb{C}, \quad s_{e}(t)=A(t) e^{i .(\omega(t) t+\phi(t))}
\end{aligned}
$$

with $A(t)$, the signal magnitude, $\omega(t)$, the frequency and $\phi(t)$ the phase shift.

For the FSK modulation with 2 levels, the signal equation becomes, without the carrier frequency:

$$
\begin{array}{lc}
\text { in } \mathbb{R}, & s_{e}(t)=A \cdot \cos \left(2 \pi\left(\alpha_{k} \cdot f_{1}+\left(1-\alpha_{k}\right) \cdot f_{2}\right) t\right) \\
\text { in } \mathbb{C}, & s_{e}(t)=A \cdot e^{i\left(2 \pi\left(\alpha_{k} \cdot f_{1}+\left(1-\alpha_{k}\right) \cdot f_{2}\right) t\right)}
\end{array}
$$

with $A$, the signal magnitude, $f_{1}$, the first level of modulation, $f_{2}$, the second level of modulation, $T$, the signal period, $\alpha_{k}$, respectively 0 when $0 \leq t<\frac{T}{2}$ and 1 for $\frac{T}{2} \leq t<T$.

2) The reception module: It mixes all contributions returned to the antenna from the scene by the wave propagation channel. The obtained mixed signal $o(t)$ corresponds to the signal received by the antenna. It is a generic module, available for all kind of antenna and modulation.

As for the transmission module, an experiment is necessary to determine the simulated noise level to add to the received signal observed in the real antenna.

3) The processing module: It contains the signal processing algorithms used to extract each target distance and speed. This module depends also on the type of modelised antenna. The results in section III are based on a simple FSK processing that consists to sample the received signal in order to separate the two 'emitted' modulation (see 2). After sampling we can extract from FFT transform the velocity for instance:

$$
v=\frac{\lambda f}{2}
$$




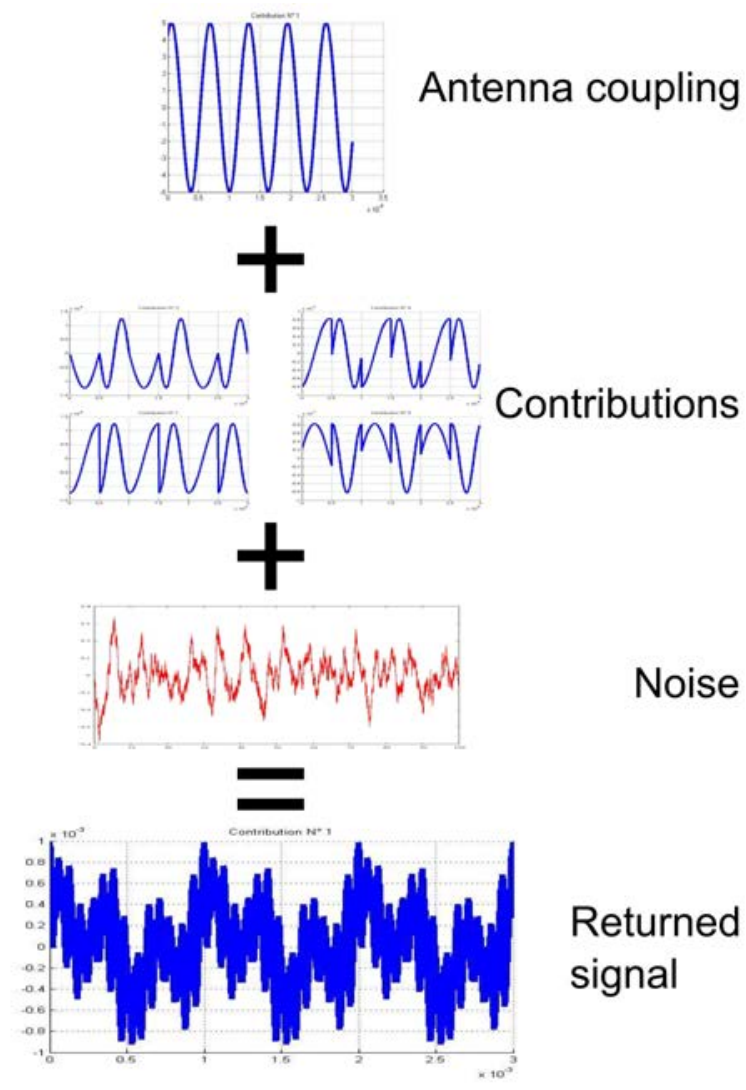

Fig. 2. Mixing procedure to retrieve the returned signal

where $f$ is a Doppler frequency. The distance is obtained with

$$
d=\frac{c \Delta \phi}{4 \pi \Delta f}
$$

where $\Delta \phi$ is the phase shift and $\Delta f$ the frequency difference due to modulation.

Depending on the resolution used to generate the signal, and in order to improve both resolution and accuracy, the sampling algorithm offers the possibility to oversample the signal to proceed.

From the previous equations, the antenna modeling is now set. The next section introduce the propagation of electromagnetic waves inside virtual scenes, that constitutes the second part of the modeling.

\section{The propagation channel modeling}

How we can simulate the propagation wave phenomenon ? To answer this question consists to ask what do we know on the sensor simulator ? SiVIC provides a lot of information about environment such as weather condition, and object properties (material, dynamic, shape, ...). Among all available informations, only some are relevant for the radar simulation and the SiVIC's architecture is capable to include additive informations on the radar model.

1) Scene modeling:

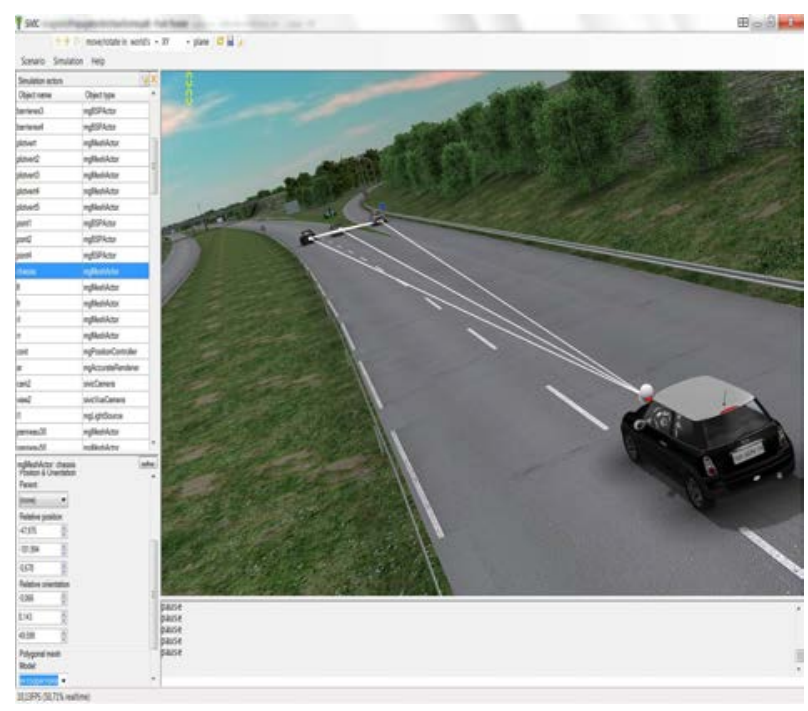

Fig. 3. An peri-urban scene used for ADAS applications

a) Electromagnetic waves representation: For the SiVIC point of view, electromagnetic waves (EW) are supposed to be plane and monochromatic. Hence, the optical properties of the electromagnetic waves can be manipulated in order to propagate the signal through the scene. Theplane and monochromatic hypothesis are considered as valid when the signal is sufficiently far from the transmitting antenna. In this case, propagation can be considered as linear, and this sounds realistic enough.

An electromagnetic wave can be interpreted in several ways, depending on how the phenomenon is considered.

Physics representation

The electromagnetic wave is modelized by an electrostatic oscillating dipole [6] defined by an electromagnetic field $E$ and $B$ moving in the direction $k$. Variations of electric and magnetic fields are related to Maxwell's equations and $(k, E, B)$ is a direct trihedron. $E$ can be expressed as $E(p, t)=$ $E_{0} e^{i .(\omega t-k p+\phi)}$, with $E_{0}$ the signal magnitude for $t=0$, $\omega=\frac{2 . \pi . c}{\lambda}$ the pulsation, $\lambda$ the wavelength, $p$ the position where the field is evaluated, and $\phi$ the phase at $t=0$.

Interactions between the EM waves and the environment follow the Maxwell's equations.

O Discrete interpretation

An electromagnetic wave can not be seen as a global effect but as a particle system [11], where particles are photons which stored the signal energy. In a monochromatic electromagnetic waves, photons are characterized by their energy $E=\hbar \omega=\hbar v=\frac{h c}{\lambda}$ and their impulsion $p=\hbar k$, with $\hbar=\frac{h}{2 \pi}$, the reduced Planck constant, $\omega=\frac{v}{2 \pi}$ the angular frequency, and $k$ the wave vector, with $|k|=\frac{2 \pi}{\lambda}$.

In practice, a given amount of photon is released in the virtual scene and the received signal energy is defined by the amount of photon returned to the antenna, which should be maximized.

The efficiency of this representation is not well-suited for the scale of automotive applications, the risk to never get 
enough returned photon is too high for real time usage.

$\bigcirc$ Continuous interpretation

The electromagnetic wave is only considered as a wave propagation phenomenon and obey to the d'Alembert equation [5]. In this representation, electromagnetic considerations are secondary and the propagation is subject to disturbing phenomenons such as reflexion, refraction, diffusion, diffraction and interferences.

The third representation was selected in this paper: computer graphics techniques can be addressed to optimize signal propagation in the scene.

b) Object representation: As well as for the representation of electromagnetic waves, different representations are possible for the scene entities. Objects can be considered as an unique entity or as a system of sub-object. For example, a car can be considered as one object or can be decomposed into the chassis and the wheels. Moreover wheels can be splitted into tire and rim and so on. Once the correct object scaling is set, the object properties must be defined. They depend on the object resolution. On high resolution, the material properties of each simple object can be used. But on low resolution, an object is composed of many materials and its characteristics can not be defined by basic physical properties but as a function of them. Finally, the geometry of the object will be considered: is it possible or not to deform an object ? therefore its geometry and its response to the electromagnetic wave.

In this work we used a mixed representation to improve the computation performances: First, all objects are not taken into account for the wave propagation, only the dynamical entities are points of interest. The environment which is static has no impact on the radar modeling. For object of interest, a point is defined, which represents the object. The object response will be defined as a function of both the incident angle and the reflection angle. Two kind of object response have been defined for these points of interest. $\bigcirc$ With lobe modeling

The first representation deals with a definition by lobes for object response. As shown on figure 4, interesting objects are modelised as ponctual source in the scene with response defined by a parametric lobe. It is the same principle used to represent lobe antenna. For an incident angle $\theta$, the para-

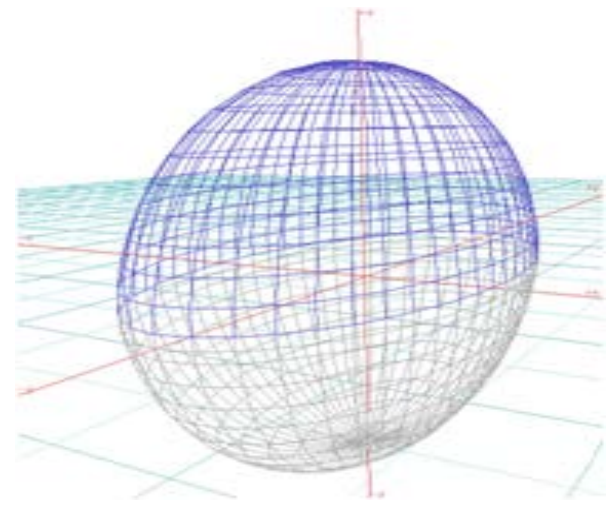

Fig. 4. Object representation with lobes metric lobe determined the gain obtained for the reflection angle $\phi$ thanks to a function $f(\theta, \phi)$. This representation is simple but doesn't provide a realistic approximation of the object response. But it is a low complexity solution which provides quite good results if properly configured.

\section{$O$ With RCS response}

The second representation is based on Radar Cross Section (RCS). A 3D record of the electromagnetic object response is used as abacus. It is obtained either by records with a real material or by computation with material models.

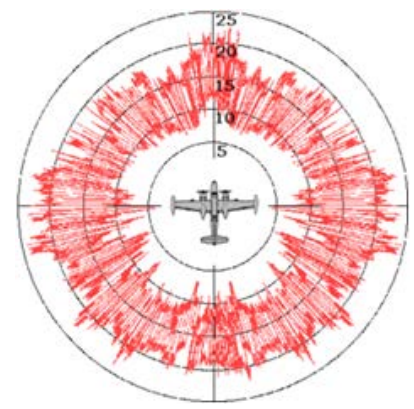

Fig. 5. Object representation with RCS

2) Wave propagation mechanism: To minimize the computation time, propagation is assumed by a ray tracer where targets are the interest points that represent the objects of the scene. The rest of the scene is used as occlusion between these nodes. Rays are casted from the transmitting antenna to the points of interest. For each reflection in the scene, rays accumulate deformations that correspond to the interactions with the objects.

3) Construction of the received signal: The contribution signals are obtained by adding deformations to the emitted signal according to the distance done by the electromagnetic waves in the environment. The deformations that are taken into account are the attenuation, the Doppler effect and the phase shift provided by the propagation channel.

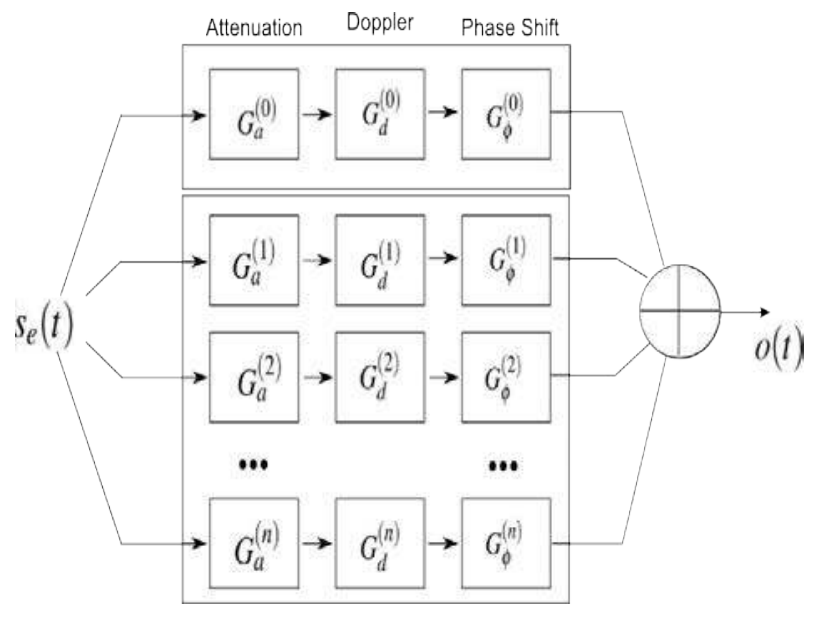

Fig. 6. Receiver Bank Filter

a) The bank filter: Each filter, $G_{a}^{(i)}, G_{d}^{(i)}$ and $G_{\phi}^{(i)}$, $i=0, \ldots, n$ deals with one aspect of the deformation applied 
to the signal. The Heaviside function used in filters is defined as $h(t-k T)$, with $k \in \mathbb{N}$ and $T$ the period.

$G_{a}^{(i)}$ is the filter associated to the object reflection attenuation.

$$
G_{a}^{(i)}(t)=\operatorname{gain}_{i} \cdot h(t-k T)
$$

where gain is obtained according to the object representation. With the RCS representation, the gain value is extracted from abaqus.

$G_{d}^{(i)}$ is the filter associated with the Doppler effect.

$$
G_{d}^{(i)}(t)=e^{2 j \pi f_{\text {Dop }_{i}} t} h(t-k T)
$$

where $f_{d o p}=\sqrt{\frac{c-\Delta v}{c+\Delta v}} f_{e}, c$ is the light velocity, $\Delta v$ the speed difference between the antenna and a mobile entity (carsq, walkers, ...). As we manipulate electromagnetic waves, we use the Doppler relativist formula, where emitter and receiver have a symetric function and where only the difference of velocities is efficient.

$G_{\phi}^{(i)}$ is the filter associated to the wave trip length.

$$
G_{\phi}^{(i)}(t)=e^{\frac{4 j \pi f_{e} d_{i}}{c}} h(t-k T)
$$

where $f_{e}$ the modulation, $d$ the distance traveled by the wave, $c$ the light celerity and $h(t-k T)$ the Heaviside function.

The phase shift of the signal is defined from the distance traveled made by the wave inside environment.

b) Rebuilding of the received signal: Once all contributions have been computed and all filters defined, the received signal can be computed as

$$
o(t)=\Sigma_{i=0}^{n} G_{a}^{(i)}(t) * G_{d}^{(i)}(t) * G_{s}^{(i)}(t) * s_{e}(t)
$$

The value $i=0$ corresponds to the coupling of the emitting and receiving antenna, $i \in[1, n]$ is the $i^{t h}$ returned contribution from the scene to the receiver.

The received signal is then transmitted to the unit of computation, which have for main task the extraction of information about targets.

\section{RESULTS}

The simulator have been tested on a computer equipped with an (c) Intel i7 $2600 \mathrm{~K} 3.40 \mathrm{GHz}$ (4 cores) and two graphical cards (c) nVidia Quadro 4000.

\section{A. Test parameters}

The radar antenna model is configured on usual frequencies available for the automotive field, i.e. a carrier frequency of $77 \mathrm{GHz}$. The FSK modulation usedare calibrated at $1 \mathrm{MHz}$ and $2 \mathrm{MHz}$. The sampling rate used for the signal generation is about $200 \mathrm{~Hz}$, and the signal duration is $1 \mathrm{~ms}$, which corresponds to an handled vector of dimension 200,000. The urban test scene involves three objects defined as targets for radar (see Fig. 3).

For the antenna modeling, the application of filters is realized independantly on each vector component, allowing parallelized processings. 3 computation methods have been implemented: $(i)$ all processing on a single processor CPU, (ii) parallelized processing on CPUs, (iii) CUDA technology for remote processing on a dedicated graphic processor (GPU).

\section{B. Performances}

The results presented in Table I were obtained from replays of the propagation channel outside the SiVIC platform in order to determine antenna model performance. The performance on the propagation channel have no significant influence on the current performance of the simulation. The computation time obtained with our architecture is below 10 ms.

\begin{tabular}{|c|c|c|c|}
\hline \multirow{3}{*}{ Antenna } & Mono-CPU & Multi-CPU & GPU \\
\cline { 2 - 4 } & $443 \mathrm{~ms}$ & $185 \mathrm{~ms}$ & $90 \mathrm{~ms}$ \\
\hline \multicolumn{4}{|c|}{ TABLE I }
\end{tabular}

Time Consumption for Antenna Model

The values in table I are signal means duration, computed on about 1500 run tests. The multi-core is not 4 times better than the mono-core since the processings cannot be totally parallelised especially the processing algorithm. The same observations can be done for the GPU version for which the data transfer time between RAM and VRAM (video memory) is added.

The observed results are comparable to the outputs from SIVIC, indicating that our model is efficient; the $10 \mathrm{~Hz}$ working frequency look like optimal for the implementation, which is equivalent to a ratio of $1 \%$ for the continuous radar signals generation. Indeed, a $1 \mathrm{~ms}$ signal needs approximately $100 \mathrm{~ms}$ of simultation.

On the other hand, if we consider only the access to information on targets, it is not necessary to generate small sample time signals. A single point for each half period is sufficient to carry out the processing. This simplification allows a drastic time reduction. And no optimisation is necessary in that case: the three computation methods provide roughly the same results. The total time consumming is approximately $43 \mathrm{~ms}$ for the whole model.

\section{Accuracy}

The last qualitative criterion is the accuracy of the target detection. The Table II presents the results obtained for a 3 cars scenario with the ground truth and the output of the radar simulation. As shown, results about target information

\begin{tabular}{|c|c|c|c|c|}
\hline \multirow{2}{*}{} & \multicolumn{2}{|c|}{ distance } & \multicolumn{2}{c|}{ velocity } \\
\cline { 2 - 5 } & theoretical & simulated & theoretical & simulated \\
\hline 1 & $30 \mathrm{~m}$ & $28.95 \mathrm{~m}$ & $-100 \mathrm{~km} / \mathrm{h}$ & $-97.35 \mathrm{~km} / \mathrm{h}$ \\
2 & $30 \mathrm{~m}$ & $30.07 \mathrm{~m}$ & $8 \mathrm{~km} / \mathrm{h}$ & $8.21 \mathrm{~km} / \mathrm{h}$ \\
3 & $30 \mathrm{~m}$ & $30.22 \mathrm{~m}$ & $25 \mathrm{~km} / \mathrm{h}$ & $22.28 \mathrm{~km} / \mathrm{h}$ \\
\hline
\end{tabular}

TABLE II

THEORIC AND SIMULATED VALUES

are consistent, but we need to keep in mind that the obtained results depend of the associated processing algorithm. The output generation processing used here is simple and results are provided from a restricted model. 


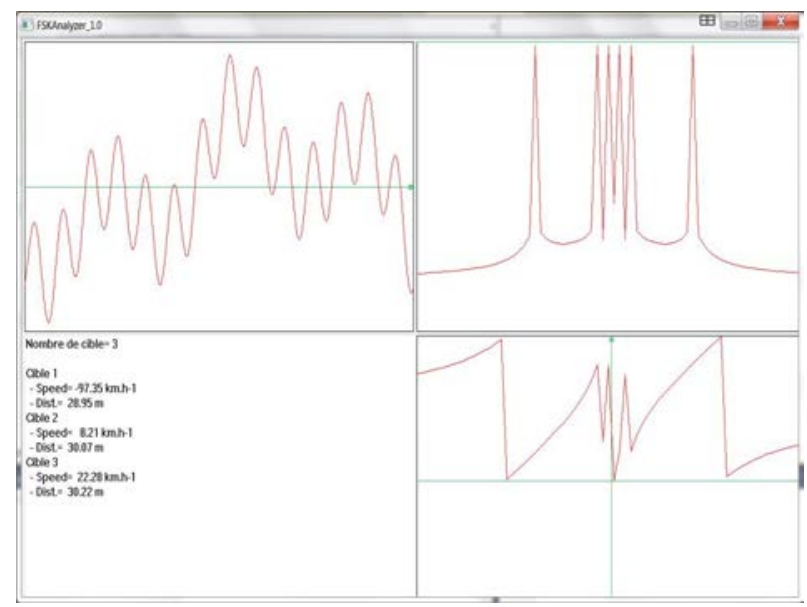

Fig. 7. Signal Analyzer for Virtual Antenna

Figure 7 provides differents view of the simulated signal. On top left corner is represented the reconstructed signal. On its right appears the Fourier Transformation of the signal and on bottom right corner is displayed phases for FFT transform. The last quarter (on bottom left corner) provides extracted information on targets.

\section{Available Scenario}

Since it is not yet possible to generate continuous signal during a real-time scenario with high sampling rate, several work modes are possible depending on whether the simulator is used for :

- ADAS prototyping: the model generates low sampled signals to obtain a real-time behavior. The generated parts of the signal are those necessary to extract the target information.

- signal analysis: 2 solutions are yet available, either a no real time generation of highly sampled signal, either an off-line reconstruction of the signal. The first solution offer the possibility to replay the scenario for the tprototyping.

- both ADAS prototyping and signal analysis: it is possible to operate in simulated time, and thus ensure the continuity of time in the scenario. In this configuration, and according to results presented below, generation of 1 second of data consumes about $1,000 \times 200=$ $200 \mathrm{sec} \approx 3 \mathrm{mns}$. It is the most time consuming mode but it allows a precise analysis of the scene.

\section{CONCLUSION}

In this paper, we have proposed the first version of radar modeling for automotive application. After a set of tests, with different configurations, the presented version provide good enough result in order to be used for ADAS prototyping. In a first step, the embedded applications identified to use simulated radar signal could be ACC, Stop and Go and interdistance regulation. For the ADAS development, without generating high-sampled signal, the current solution is ready for real-time computation and provides satisfying results about environment perception. Actually, only one model of radar antenna has been implemented but the architecture design provide an easy integration way for future models. About the signal generation, it is not yet possible to generate a continous signal in real time. A lot of mechanisms are available to obtain them and be able to inject results in replayed ADAS scenario test. This work also allows to highlight some limitation of the model. One issue is about the RCS modeling. The associated RCS table are too huge to be considered as a viable solution, and lobe definition is not realistic, even if results are closed to the expected ones. But even if improvment are planned, this radar simulation will stay a good tool for modest development platform and signal post processing and analysing.

\section{FUTURE WORKS}

As explained in the conclusion, a lot of improvement have to be realised to obtain the desired sensor model in terms of realism. The work presented in this article have been realised for the french project named eMotive and will continue in a collaborative project between France and Canada, named CooPerCom. Both, antenna model and propagation channel model, must be improved. New type of antenna have to be implemented, such as Fequency Modulated Continuous Wave (FMCW), to be able to detect static target. About the propagation channel, a new version, based on material properties and which take int account all the environment is actually in development. It uses massive parallelization thanks to CUDA technology.

And even if results are hopeful, models need to be validate with rigourous statistical methodology. A campaign of data collection should be carried out this year with an oem sensor to validate the model for at least one manufactured sensor.

\section{REFERENCES}

[1] H.J. Mametsa A. Berges, J. Latger. Fermat a new radar simulation approach. RADAR, June 2004.

[2] ANR. Endocom. "http://www.endocom.upme.fr/".

[3] ANR. Multipass. "http://multipass.elibel.tm.fr/".

[4] ANR. Op2h. "http://op2h.univ-mlv.fr/liens.html".

[5] J. Cooper. Long-time behavior and energy growth for electromagnetic waves reflected by a moving boundary. Antennas and Propagation, IEEE Transactions on, 41(10):1365 -1370, oct 1993.

[6] D. S. Jones. Acoustic and electromagnetic waves. 1986.

[7] Yahya S. H. Khraisat. Simulation of the $24 \mathrm{ghz}$ short range, automotive radar. European Journal of Scientific Research, 67:75-82, 2011.

[8] LIVIC. Website of the professional version of sivic. "http: / / www . civitec.com/".

[9] Z. Mahfouz, A. Gati, Man-Fai Wong, J. Wiart, D. Lautru, and V.F. Hanna. Statistical analysis of real radiofrequencies exposure in a realistic environment. In Microwave Symposium (MMS), 2010 Mediterranean, pages 342 -345, aug. 2010.

[10] R. Moghrani, J.M. Conrat, X. Begaud, and B. Huyart. Performance evaluation of a $3 \mathrm{~d}$ ray tracing model in urban environment. In Antennas and Propagation Society International Symposium (APSURSI), 2010 IEEE, pages $1-4$, july 2010.

[11] S.A. Schelkunoff. Electromagnetic waves. Van Nostrand Reinhold, 1943 